\title{
ChemComm
}

Check for updates

Cite this: Chem. Commun., 2018 54, 13355

Received 19th July 2018,

Accepted 3rd October 2018

DOI: $10.1039 / \mathrm{c} 8 \mathrm{cc} 05899 \mathrm{~g}$

rsc.li/chemcomm

\section{Size-controlled and water-soluble gold nanoparticles using UV-induced ligand exchange and phase transfer $\dagger$}

\author{
Jan Bart ten Hove, (D) $\ddagger^{a}$ Laura M. I. Schijven, (D) $\ddagger^{a b}$ Junyou Wang (D) and \\ Aldrik H. Velders (D) *a
}

\begin{abstract}
Oleylamine-capped gold nanoparticles (AuNPs) with sizes ranging from 5 to $13 \mathrm{~nm}$ and narrow size distributions (<10\%) are synthesized by using a seeded growth approach. Water-solubility is achieved by using a UV-induced ligand exchange approach, resulting in transfer from the organic to an aqueous phase.
\end{abstract}

Gold nanoparticles (AuNPs) are widely applied because of their characteristic size-dependent physical and chemical properties. ${ }^{1-5}$ Some of their most important applications include staining in electron microscopy, ${ }^{6,7}$ catalysis, ${ }^{8}$ and biosensing. ${ }^{9-14}$ Many of these applications - particularly the biological ones - need well-defined, water-dispersed AuNPs. ${ }^{15}$ The most well-known method to synthesize water-dispersed AuNPs was developed by Turkevich in $1951 .{ }^{16}$ Unfortunately, this method has inherent drawbacks such as the stability of AuNPs and the broad size distribution $(>10 \%)$ of smaller $(<20 \mathrm{~nm})$ AuNPs. ${ }^{17}$ Synthesizing AuNPs in an organic solvent, on the other hand, allows more control over the nanoparticle size and narrow size distributions $(<10 \%)$ compared to similar-sized AuNPs synthesized in an aqueous phase. ${ }^{1,17-20}$ The most widely used strategy to synthesize AuNPs in an organic solvent is the Schiffrin-Brust method. ${ }^{21}$ This method yields AuNPs with a narrow size distribution using a strong coordinating thiol ligand. ${ }^{18,22,23}$ Peng et al. developed a method to synthesize $5 \mathrm{~nm}$ AuNPs with a narrow size distribution, by using a weakly coordinating oleylamine ligand. ${ }^{8}$ However, as these AuNPs are exclusively soluble in organic solvents, their application in aqueous, e.g. biological, systems is restricted. Various methods have been developed to render hydrophobic nanoparticles water soluble, such as ligand exchange. ${ }^{24-28}$ An interesting variation of this method is the UV-induced in situ ligand exchange and phase transfer approach as reported by the group of Mattoussi. Using this approach, the native, hydrophobic

\footnotetext{
${ }^{a}$ Laboratory of BioNanoTechnology, Wageningen University, Bornse Weilanden 9, 6708 WG Wageningen, The Netherlands. E-mail: aldrik.velders@wur.nl

${ }^{b}$ Laboratory of Biobased Chemistry and Technology, Wageningen University, Bornse weilanden 9, 6708 WG Wageningen, The Netherlands

$\dagger$ Electronic supplementary information (ESI) available. See DOI: 10.1039/c8cc05899g \$ Equal contribution.
}

and weakly coordinating ligand on semiconductor nanoparticles is replaced by a hydrophilic, multicoordinating lipoic acid derivative. These quantum dot nanoparticles then transferred from organic to aqueous solvents, and showed excellent colloidal stability. ${ }^{3,29,30}$ We now show that oleylamine-capped AuNPs of well-defined and tunable size can be transformed into water-soluble gold nanoparticles of identical size by applying the UV-induced ligand exchange approach.

Here, we report a strategy to obtain water-soluble AuNPs with sizes ranging from 5 to $13 \mathrm{~nm}$ and narrow size distributions $(<10 \%)$. Our strategy is as follows (Scheme 1): $4.5 \mathrm{~nm}$ oleylamine-capped AuNP seeds were synthesized using Peng et al.'s method. ${ }^{8}$ Subsequently, these AuNPs were used in a seeded growth reaction to obtain sizes of 7-13 $\mathrm{nm}$. The AuNP phase transferred quantitatively to the aqueous phase upon UV-induced photoligation of lipoic acid..$^{29}$ The process of phase transfer was indicated by the change of color of the bottom aqueous layer to deep-red and the completion by the loss of color of the top organic layer.

The AuNPs produced using the strategy presented in Scheme 1 are shown in Fig. 1. Starting from $4.5 \mathrm{~nm}$ oleylaminecapped AuNP seeds, we obtained AuNPs with sizes ranging from 7 to $13 \mathrm{~nm}$ (A-E) via a seeded growth approach. Seeded growth reactions rely on the slow reduction and deposition of a gold precursor on a seed, resulting in controlled growth of the seeds. ${ }^{31}$ Transmission electron microscopy (TEM) images showed that the AuNPs form large, hexagonally ordered crystals when deposited on a TEM grid, indicating their narrow size distributions. ${ }^{31}$ This infers that the narrow size distribution of the seeds is retained throughout the seeded growth approach (Fig. S1, ESI $\dagger$ ). The UV-Vis absorbance spectra of these AuNPs showed sharp localized surface plasmon resonance (LSPR) peaks with peak maxima that characteristically redshift for bigger particles, from $513 \mathrm{~nm}$ (seeds) to $530 \mathrm{~nm}(\mathrm{E}) .^{8}$ DLS characterization of the seeds and samples A-E showed the absence of aggregates in the correlation curves (Fig. S2, ESI $\dagger$ ), and by fitting the data, we found for all samples that the sizes were in concordance with the TEM analysis and the UV-Vis measurements (Fig. S1, ESI $\dagger$ ). 


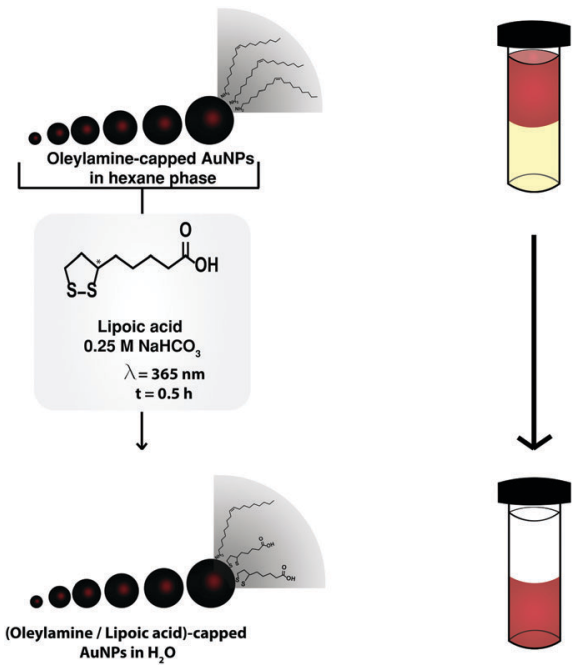

Scheme 1 Schematic representation of UV-induced phase transfer of hydrophobic AuNPs. AuNPs, suspended in an organic phase, are mixed with an aqueous solution of lipoic acid in $0.25 \mathrm{M} \mathrm{NaHCO}_{3}$. Upon UVirradiation for 30 minutes, the AuNPs quantitatively phase-transfer from the organic to the aqueous layer.

Next, these samples were subjected to the UV-induced ligand exchange and phase transfer. The AuNPs quantitatively phase transferred from the organic phase to water, which generally proceeded in less than 30 minutes. We do note here that with increasing AuNP size, the time to achieve complete phase transfer also increased from minutes to about an hour. TEM on these AuNPs after phase transfer (seeds ${ }^{\prime}$ and $\mathrm{A}^{\prime}-\mathrm{E}^{\prime}$ ) showed that the sizes and size distributions of the samples were comparable to those before ligand exchange (Fig. S3 and Table S1, ESI $\dagger$ ). Characterization via UV-Vis spectroscopy showed the same trend as before phase transfer, with a slight redshift of the LSPR peak ( $\sim 3 \mathrm{~nm}$, Table S1, ESI $\dagger$ ), which is attributed to the differences in solvent polarity and capping ligand. ${ }^{32,33}$ Finally, DLS characterization (Fig. S4, ESI $\dagger$ ) showed the absence of aggregates. The measured diameters were $\pm 1 \mathrm{~nm}$ smaller after phase transfer and we attribute this to the differences in size of the ligands. Finally, ${ }^{1} \mathrm{H}$ nuclear magnetic resonance (NMR) was used to analyze the surface composition of the AuNPs, after seeded growth and ligand exchange $\left(\mathrm{A}^{\prime}-\mathrm{E}^{\prime}\right)$. The AuNPs were digested using $\mathrm{KCN}$ to release surface-bound ligands into solution, which eliminates effects of severe line broadening in the ${ }^{1} \mathrm{H}$ NMR spectra. ${ }^{34-37}$ For relative ligand quantification, the integrated peak intensity associated with lipoic acid ( $\delta=3.5-3.2 \mathrm{ppm})$ was compared to the one of oleylamine $(\delta=1.15-0.85 \mathrm{ppm}) .{ }^{37}$ These spectra showed that, after ligand exchange, the AuNPs are capped with lipoic acid with some (9-14\%) residual oleylamine still present (Fig. S5 and Table S2, ESI $\dagger$ ). We have also applied this method to sample $\mathrm{A}^{\prime}$ with longer reaction times for the exchange and phase transfer. The spectra showed that increasing the exchange reaction time does not affect the amount of residual oleylamine on the AuNP surfaces (Table S3, ESI $\dagger$ ).

AuNPs find applications in a plethora of areas, including detection of analytes via aggregation of multivalent networks. ${ }^{10-13}$ Here we present the sensing properties of the water-soluble AuNPs showing a color change from red to purple, corresponding to a red shift in optical absorption, upon aggregation
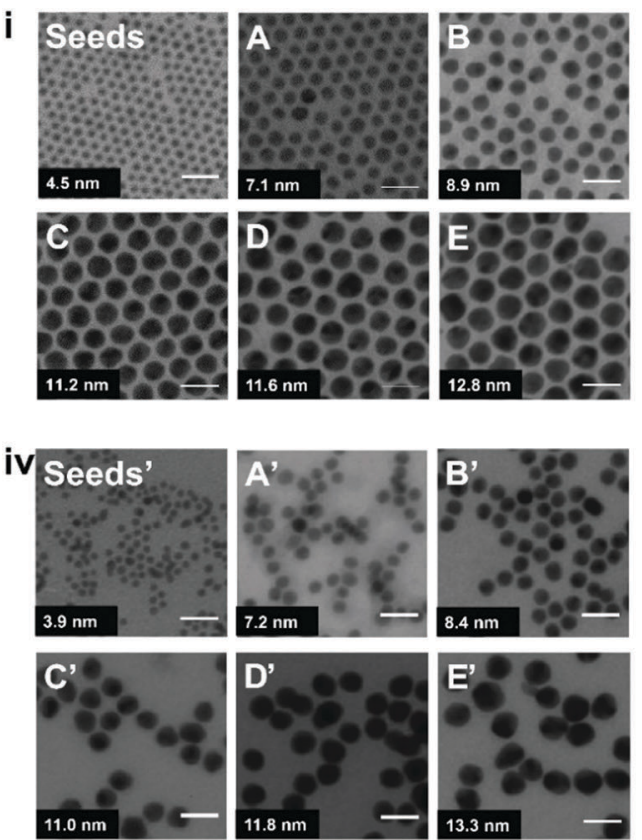

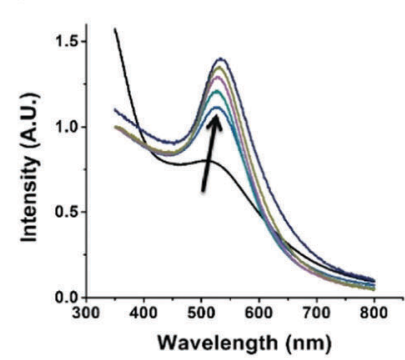

iii
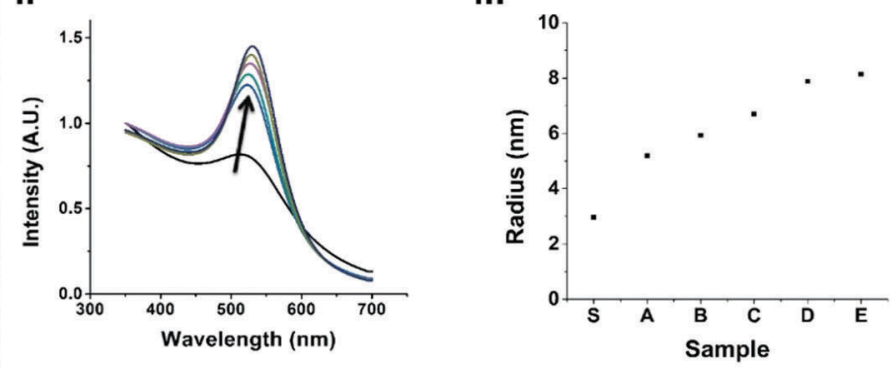

vi

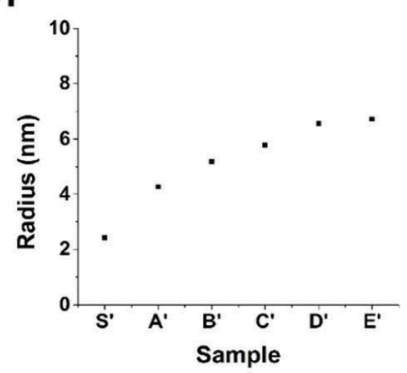

Fig. 1 UV-induced ligand exchange and phase transfer of AuNPs from (i-iii) organic to (iv-vi) aqueous phase. (i and iv) TEM images of AuNP seeds and AuNPs obtained after the seeded growth (A-E). (iv) The respective seeds (seeds') and samples $\left(\mathrm{A}^{\prime}-\mathrm{E}^{\prime}\right)$ after phase transfer. Scale bars are $20 \mathrm{~nm}$. (ii and $v$ ) Normalized UV-Visible absorption spectra before (ii) and (v) after phase transfer, showing a clear red shift of the LSPR peaks. The spectra are normalized at $\lambda=350 \mathrm{~nm}$ and are shifted vertically to facilitate comparison between the samples. (iii and vi) Hydrodynamic radii of the AuNPs, as determined from number-averaged DLS fits of the AuNPs (iii) before and (vi) after ligand exchange. 

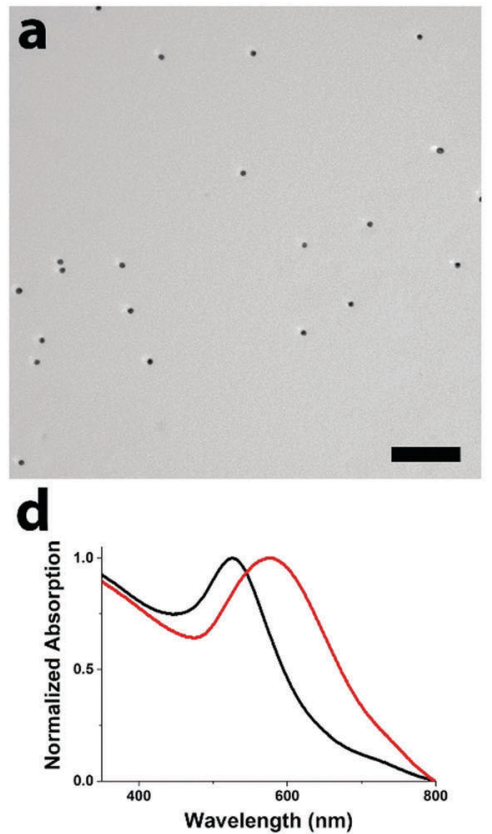

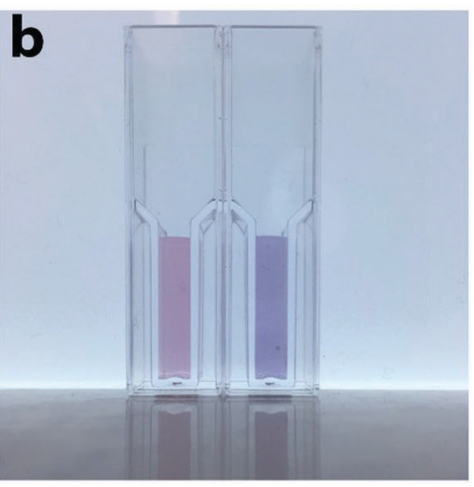

e

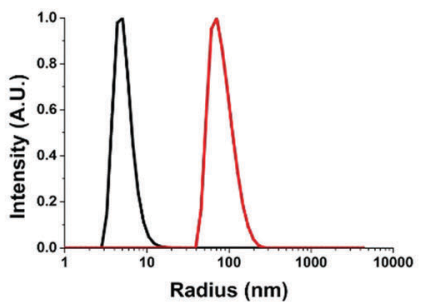

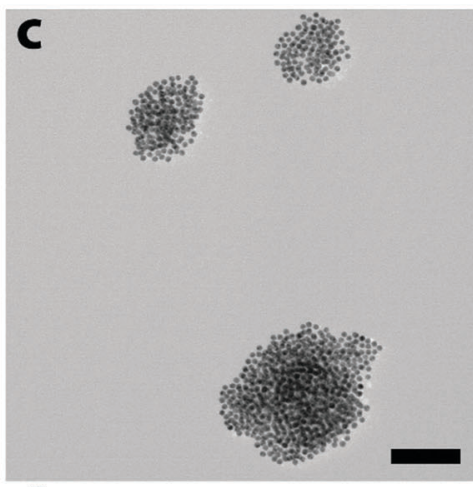

f

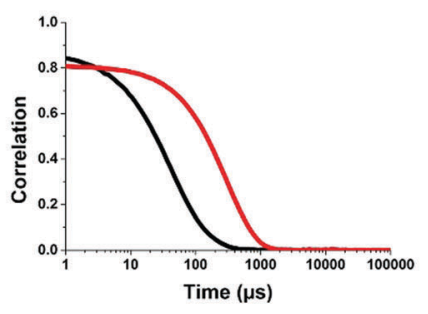

Fig. 2 Formation of PAMAM-AuNP composite upon the addition of $\sim 3$ nmoles of PAMAM-G5-NH $\mathrm{N}_{2}(+)$ to a solution containing $\sim 3 \mathrm{nmoles}$ of AuNPs (-). (a) TEM micrograph of suspended sample $B^{\prime}$. (b) Photograph showing $B^{\prime}$ without (left) and with (right) PAMAM-G5-NH ${ }_{2}$. After the addition of PAMAMG5- $\mathrm{NH}_{2}$ the color of the solution changes from red to purple. (c) TEM micrograph of the formed PAMAM-AuNP composite, showing that extended

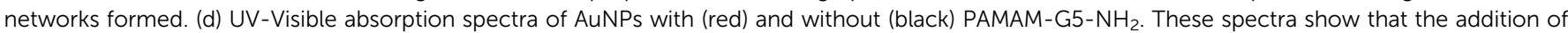
PAMAM-G5- $\mathrm{NH}_{2}$ results in a redshift of the LSPR peak. (e and f) DLS characterization of AuNPs with (red) and without (black) added PAMAM-G5-NH 2 . The number-weighed size fit (e) indicates that the AuNP hydrodynamic radius is $\sim 5 \mathrm{~nm}$, whereas the hydrodynamic radius of the PAMAM-AuNP composite is $\sim 80 \mathrm{~nm}$. The DLS correlation curve (f) shows that the addition of PAMAM-G5- $\mathrm{NH}_{2}$ results in an increase of the correlation time, indicative of the formation of larger particles. Scale bars in (a) and (c) represent $100 \mathrm{~nm}$.

with amine-terminated fifth generation poly(amidoamine), PAMAMG5, dendrimers (see Fig. 2). ${ }^{38}$ The formation of multivalent networks is further corroborated by TEM and DLS measurements. Control experiments with the addition of water, salt, and negatively and positively charged PAMAM, and in buffer (see the ESI, $\uparrow$ S3) prove the selectivity of these AuNPs for positively charged PAMAM.

Here we showed a method to prepare size-controlled and watersoluble AuNPs. $4.5 \mathrm{~nm}$ oleylamine-capped AuNP seeds were synthesized in an organic solvent. The AuNP sizes were then tuned from 4.5 to 7-13 nm via a seeded growth reaction approach, while retaining the narrow size distributions. By combining these oleylamine-capped AuNPs with UV-induced photoligation of lipoic acid, we achieved quantitative phase transfer of the AuNPs to the aqueous phase, proceeding due to ligand exchange of oleylamine for lipoic acid. We believe that the here reported strategy is a straightforward and robust approach to transfer AuNPs from organic to aqueous solvents. This approach can be applied to obtain well-defined AuNPs in high concentrations, for use in studies where control over the nanoparticle size, size distribution and surface composition is of importance.

JBtH and AHV thank NWO for financial support of the MONALISA project 717.013.006. We thank the Wageningen Electron Microscopy Centre for their support with the TEM measurements.

\section{Conflicts of interest}

There are no conflicts to declare.

\section{Notes and references}

1 P. Zhao, N. Li and D. Astruc, State of the art in gold nanoparticle synthesis, Coord. Chem. Rev., 2013, 257(3-4), 638-665.

2 W. Maria, et al., Turkevich in New Robes: Key Questions Answered for the Most Common Gold Nanoparticle Synthesis, ACS Nano, 2015, 9(7), 7052-7071.

3 N. Zhan, et al., Controlling the Architecture, Coordination, and Reactivity of Nanoparticle Coating Utilizing an Amino Acid Central Scaffold, J. Am. Chem. Soc., 2015, 137(51), 16084-16097.

4 M.-C. Daniel and D. Astruc, Gold nanoparticles: assembly, supramolecular chemistry, quantum-size-related properties, and applications toward biology, catalysis, and nanotechnology, Chem. Rev., 2004, 104(1), 293-346.

5 Y. Xia, et al., Shape-controlled synthesis of metal nanocrystals: simple chemistry meets complex physics?, Angew. Chem., Int. Ed., 2009, 48(1), 60-103.

$6 \mathrm{~J}$. Roth, The silver anniversary of gold: 25 years of the colloidal gold marker system for immunocytochemistry and histochemistry, Histochem. Cell Biol., 1996, 106(1), 1-8.

7 W. P. Faulk and G. M. Taylor, An immunocolloid method for the electron microscope, Immunochemistry, 1971, 8(11), 1081-1083.

8 S. Peng, et al., A facile synthesis of monodisperse Au nanoparticles and their catalysis of CO oxidation, Nano Res., 2008, 1(3), 229-234.

9 S. Zeng, et al., Nanomaterials enhanced surface plasmon resonance for biological and chemical sensing applications, Chem. Soc. Rev., 2014, 43(10), 3426-3452.

10 C. Robert, et al., Multivalent Nanoparticle Networks Enable Point-ofCare Detection of Human Phospholipase-A2 in Serum, ACS Nano, 2015, 9(3), 2565-2573.

11 R. de laRica, et al., Multivalent Nanoparticle Networks as Ultrasensitive Enzyme Sensors, Angew. Chem., Int. Ed., 2011, 50(25), 5704-5707.

12 R. de la Rica and A. H. Velders, Supramolecular Au Nanoparticle Assemblies as Optical Probes for Enzyme-Linked Immunoassays, Small, 2011, 7(1), 66-69.

13 X. Yang, et al., Gold Nanomaterials at Work in Biomedicine, Chem. Rev., 2015, 115(19), 10410-10488. 
14 S. Yapar, et al., Dipeptide recognition in water mediated by mixed monolayer protected gold nanoparticles, Chem. Commun., 2015, 51(75), 14247-14250.

15 X.-D. Zhang, et al., Size-dependent in vivo toxicity of PEG-coated gold nanoparticles, Int. J. Nanomed., 2011, 6, 2071-2081.

$16 \mathrm{~J}$. Turkevich, P. C. Stevenson and J. Hillier, A study of the nucleation and growth processes in the synthesis of colloidal gold, Discuss. Faraday Soc., 1951, 11, 55-75.

17 H. Hiramatsu and F. E. Osterloh, A Simple Large-Scale Synthesis of Nearly Monodisperse Gold and Silver Nanoparticles with Adjustable Sizes and with Exchangeable Surfactants, Chem. Mater., 2004, 16(13), 2509-2511.

$18 \mathrm{Y}$. Li, et al., Mechanistic insights into the brust-schiffrin two-phase synthesis of organo-chalcogenate-protected metal nanoparticles, J. Am. Chem. Soc., 2011, 133(7), 2092-2095.

19 R. Sardar and J. S. Shumaker-Parry, 9-BBN induced synthesis of nearly monodisperse -functionalized alkylthiol stabilized gold nanoparticles, Chem. Mater., 2009, 21(7), 1167-1169.

20 N. Zheng, J. Fan and G. D. Stucky, One-step one-phase synthesis of monodisperse noble-metallic nanoparticles and their colloidal crystals, J. Am. Chem. Soc., 2006, 128(20), 6550-6551.

$21 \mathrm{M}$. Brust, et al., Synthesis of thiol-derivatised gold nanoparticles in a two-phase liquid-liquid system, J. Chem. Soc., Chem. Commun., 1994, 801-802.

22 D. V. Leff, et al., Thermodynamic control of gold nanocrystal size: experiment and theory, J. Phys. Chem., 1995, 99(18), 7036-7041.

23 M. J. Hostetler, et al., Alkanethiolate gold cluster molecules with core diameters from 1.5 to $5.2 \mathrm{~nm}$ : core and monolayer properties as a function of core size, Langmuir, 1998, 14(1), 17-30.

24 I. Ojea-Jiménez, L. García-Fernández and J. Lorenzo, Facile preparation of cationic gold nanoparticle-bioconjugates for cell penetration and nuclear targeting, ACS Nano, 2012, 6(9), 7692-7702.

25 V. J. Gandubert and R. B. Lennox, Assessment of 4-(dimethylamino) pyridine as a capping agent for gold nanoparticles, Langmuir, 2005, 21(14), 6532-6539.
26 D. I. Gittins and F. Caruso, Spontaneous phase transfer of nanoparticulate metals from organic to aqueous media, Angew. Chem., Int. Ed., 2001, 40(16), 3001-3004.

27 J. Yang, J. Y. Lee and J. Y. Ying, Phase transfer and its applications in nanotechnology, Chem. Soc. Rev., 2011, 40(3), 1672-1696.

$28 \mathrm{~J}$. Hassinen, et al., Rapid Cationization of Gold Nanoparticles by Two Step Phase Transfer, Angew. Chem., Int. Ed., 2015, 54(27), 7990-7993.

29 G. Palui, et al., Photoinduced Phase Transfer of Luminescent Quantum Dots to Polar and Aqueous Media, J. Am. Chem. Soc., 2012, 134(39), 16370-16378.

30 F. Aldeek, et al., UV and Sunlight Driven Photoligation of Quantum Dots: Understanding the Photochemical Transformation of the Ligands, J. Am. Chem. Soc., 2015, 137(7), 2704-2714.

$31 \mathrm{~N}$. Goubet, et al., Size and nanocrystallinity controlled gold nanocrystals: synthesis, electronic and mechanical properties, Nanoscale, 2015, 7(7), 3237-3246.

32 S. K. Ghosh, et al., Solvent and ligand effects on the localized surface plasmon resonance (LSPR) of gold colloids, J. Phys. Chem. B, 2004, 108(37), 13963-13971.

33 E. Hutter and J. H. Fendler, Exploitation of localized surface plasmon resonance, Adv. Mater., 2004, 16(19), 1685-1706.

34 B. C. Mei, et al., Effects of ligand coordination number and surface curvature on the stability of gold nanoparticles in aqueous solutions, Langmuir, 2009, 25(18), 10604-10611.

35 D. Dorokhin, et al., Reversible Phase Transfer of (CdSe/ZnS) Quantum Dots between Organic and Aqueous Solutions, ACS Nano, 2009, 3(3), 661-667.

36 L. E. Marbella and J. E. Millstone, NMR techniques for noble metal nanoparticles, Chem. Mater., 2015, 27(8), 2721-2739.

37 A. M. Smith, et al., Quantitative analysis of thiolated ligand exchange on gold nanoparticles monitored by ${ }^{1} \mathrm{H}$ NMR spectroscopy, Anal. Chem., 2015, 87(5), 2771-2778.

38 S. Srivastava, B. L. Frankamp and V. M. Rotello, Controlled Plasmon Resonance of Gold Nanoparticles Self-Assembled with PAMAM Dendrimers, Chem. Mater., 2005, 17(3), 487-490. 